\title{
Recent Advances in synthesis and Application of Hierarchical Zeolite Zhuogi Yang ${ }^{1, \text { a }}$ \\ ${ }^{1}$ College of Chemistry, Jilin University, Changchun 130012, China; \\ azhuo7yang@126.com
}

\begin{abstract}
Keywords: Zeolite, Hierarchical pores,Diffusion,Catalytic applications.
\end{abstract}
\begin{abstract}
Constructing hierarchical Zeolite is an important method to enhance accessibility of molecular sieves and the research hotspot in catalytic field. A great number of synthetic methods for adjusting the pore structure of hierarchical molecular sieves were developed. The synthetic researches on hierarchical molecular sieves were reviewed from three different aspects as follows: demetalization, in-situ crystallization and recrystallization. Demetalization mainly included steaming treatment and chemical treatment. In-situ crystallization included hard template and soft template methods. Recrystallization was a method of synthesizing hierarchical molecular sieves, which was obtained by the alkali treatment of zeolite crystal under the existence of surfactants. In addition, the prospect of hierarchical molecular sieves was also outlined.
\end{abstract}

\section{Introduction}

Zeolites represent the most important group of industrial heterogeneous catalysts with large-scale applications in refining and petrochemistry and increasing potential in environmental catalysis and synthesis of fine chemicals $[1,2]$. Zeolites are crystalline microporous aluminosilicates offering a plethora of modifications and opportunities for further improvement of their properties $[3,4,5]$. At present, 200 different structural types have been recognized differing in the size, shape and connectivity of their channels usually being from 8 to 30 -rings $[6,7,8,9]$. The size of zeolite channel entrances sometimes overcomes $1 \mathrm{~nm}$, but it should be stressed that for industrial applications the maximum channel size is still $0.74 \mathrm{~nm}$ (12-ring). The possibility to control the textural properties together with adjustable acidity (type, concentration and strength of acid sites) is the most important feature of zeolites.

Since zeolites are crystalline aluminosilicates with three dimensional microporous structures [10]. They have been used as ion exchangers, catalysts, and adsorbents in various fields such as oil refining, petro-chemistry, agriculture, and water and wastewater treatment. Their wide use is because of their many beneficial properties, such as framework and compositional flexibilities, physical and hydro-thermal stabilities, non-toxicity, high surface areas, exchangeable cations, and good cost-benefit ratios. Although many zeolite applications depend on their microporous structures, this can cause diffusional constraints for bulky reactant and product molecules. There have been many efforts to overcome the intrinsic limitations of conventional zeolites by preparing hierarchically structured zeolites. As a result of these efforts, several strategies have been established and the use of new zeolitic materials in various catalytic and adsorptive reactions has been investigated. Longer lifetimes, high catalytic performances, and postponed coking and catalyst deactivation can be achieved using hierarchical zeolites.

Combining with the advantages of microporous molecular sieve and mesoporous material, building a new catalytic material with multi hole has become a hotspot in the field of catalysis, synthesis of hierarchical mesoporous molecular sieve to increase the diffusion properties of molecular sieve surface is the main research content. 
Synthesis of hierarchical zeolites

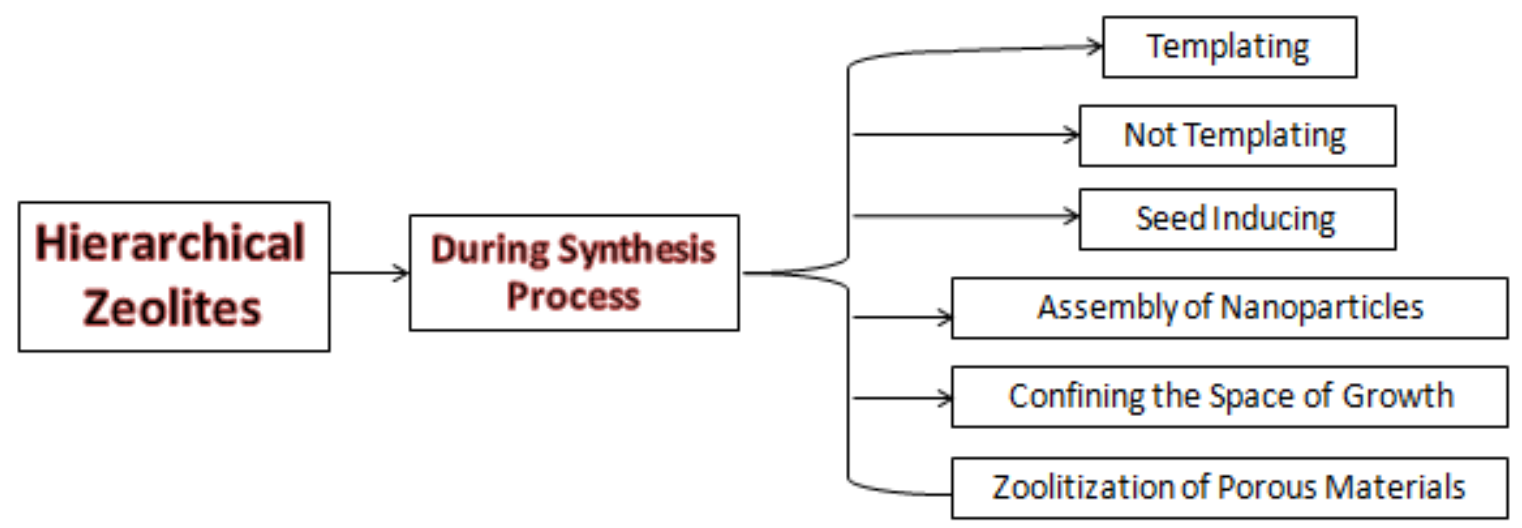

Fig.1. Overview of synthetic methods for hierarchical zeolites

Synthesis of hierarchical zeolites by demetallation. In the demetallation method, one constituent is preferentially extracted from a preformed zeolite to create secondary porosity in the structure, e.g., desilication and dealumination of the zeolite framework[6]. Dealumination is an effective method for synthesizing zeolites with large pores and has been used in industry for many years. Mineral and organic acid solutions, various chemicals, and high temperature during calcinations and steaming can be used to eliminate $\mathrm{Al}$ species from the framework. In all cases, $\mathrm{Si}-\mathrm{O}-\mathrm{Al}$ bonds are hydrolyzed to decrease the $\mathrm{Al}$ content [7]. The first step after preparing the desired zeolite is calcination, in which some $\mathrm{Al}$ atoms are released from the framework, depending on the temperature and type of zeolite. Steam treatment at high temperature is a well-established method for adjusting the acidity of a zeolite [8].

In summary, the main advantage of desilication is its simplicity and inexpensive starting materials. However, partial damage of the zeolitic structure and loss of micropores and crystallinity can occur, therefore the treatment should be precisely controlled to prevent formation of amorphous parts or extra-framework Al [9]. Pretreatment of zeolites or using low-temperature calcined zeolites with less-damaged structures and use of a surfactant to help recrystallization are rational strategies for overcoming these problems [10].

Synthesis of hierarchical zeolites by surfactant assisted recrystallization method.

Mesoporosity can be effectively introduced into zeolitic structure using mild conditions (e.g., dilute $\mathrm{Na} \mathrm{OH}$ solution, low temperature and short time) together with a cationic surfactant such as cetyltrimethylammonium bromide or chloride (CTAB or CTAC), i.e., recrystallization of microporous zeolites to micro-mesoporous ones or meso-structuring of zeolites. This process prevents dissolution of the crystals by providing interactions between the surfactant and the zeolite, and almost complete recovery is achieved as a result of reorganization of the zeolite lattice around surfactant micelles $[11,12,13]$.

To summarize, the preparation of hierarchical zeolites using surfactant assisted recrystallization is an easy industrial process that prevents the framework damage that occurs in the desilication method.

Synthesis of hierarchical zeolites by zeolitization of mesoporous/macroporous starting materials. Extensive studies have enabled the conversion of mesoporous structures with amorphous walls to crystalline zeolite frameworks. Dry gel conversion in the presence of an organic microporous SDA resulted in ordered mesoporous substances such as SBA-15 and MCM-41 undergoing partial crystallization on their walls to give a zeolitic structure. This method leads to intercrystalline mesopore formation. Clearly, the morphology, topology, and phase purity of the zeolite crystals, and the distribution, location, size, shape, and terconnectivity of the mesopores, are closely related to the template type and how well they interact with alumino silicate species in the synthesis gels [14]. Unfortunately, during conventional hydrothermal synthesis, the zeolite and mesoporous substrates nucleate synchronously, resulting in the formation of a physical mixture consisting of both components. Moreover, crystallization of mesopore walls to zeolites to form the 
resultant product, which have different crystalline and amorphous phase densities, causes breakage of the aluminosilicate framework, so partial collapse of the final meso-structured material occurs.

In another approach, careful deposition of presynthesized nanozeolite seeds onto an ordered/non-ordered macro or mesoporous support resulted in zeolitized porous materials. The method used to introduce nanozeolite seeds into mesoporous structures is clearly an important subject. Better yields are obtained by wet impregnation from a clear solution rather than mechanical mixing of both powders before calcination. However, zeolite particle deposition on the external surfaces of the porous supports can cause pore entrance blockage.

Synthesis of hierarchical zeolites by nanoparticle assembly. Mesopores in a hierarchical zeolite can be present in three forms: intercrystalline, intracrystalline, and both between inside crystals. A hierarchical zeolite with an intercrystalline mesoporous structure can be obtained by controlling the conditions to reinforce nucleation, nanozeolite preparation, and aggregation of nanozeolite seeds with voids between them. These materials give better performances than conventional zeolites because of two good features, i.e., the nanoscale zeolite crystals have much higher external surface areas and the presence of larger pores among the nanocrystals accelerates mass transport of the reactants and products [15].

Template assisted synthesis of hierarchical zeolites. The use of soft or hard meso templates in addition to microporous SDAs in conventional zeolite synthesis gels can induce additional porous systems in the zeolite framework structure. There have been many studies of the use of hard templates with zeolite synthesis gels. In the preparation of ZSM-5 nanozeolites using a confined space method, an initial product is formed by excess gel adsorption on carbon black particles at the impregnation stage. After zeolite crystal formation, combustion in air is conducted to remove the carbon template and obtain mesopores comparable to the carbon particle size [16].

\section{Catalytic applications of hierarchical zeolites}

Hierarchical zeolites have potential applications in many fields because of their properties, and various methods for their synthesis have been developed. For reactions involving the diffusion of small molecules into micropores, the results achieved using conventional and hierarchical zeolites are similar; however, reactions involving bulky substrates profit from the multimodal porosities of hierarchical zeolites [17]. Alkylation, somerization, methanol to hydrocarbons (MTH) conversion, aromatization, polymer pyrolysis, condensation, and atalytic cracking reactions are among the many types of reactions that benefit from the use of hierarchical zeolites. For example, in the alkylations of benzene with isopropanol using conventional and hierarchical $\beta$ zeolites, higher activity and selectivity for the main product (isopropylbenzene) were achieved in the presence of mesoporous zeolites, as a result of improved mass transfer and the secondary pore system [18].

As mentioned earlier, one important problem that microporous structures usually face during catalytic processes is blockage of the pore mouths as a result of coke deposition, resulting in catalyst deactivation. Coke formation is unavoidable. It poisons the catalyst and cannot be recovered, and therefore it is essential to minimize coke formation. Many studies have shown that coke formation can be avoided using the large pore systems in mesoporous zeolites. In most cases, improved catalytic performances, e.g., enhanced catalytic conversions, improved selectivities, restricted coking and catalyst deactivation, and longer catalyst lifetimes, are observed because of the auxiliary mesoporosity in the zeolite systems, which enables these materials to be used as efficient catalysts in a wide range of reactions.

\section{Summary}

Zeolite catalysts have a wide range of industrial applications, and therefore the development of zeolite catalysts that give improved yields in industrial reactions is important. There are several methods for producing nanosized and hierarchical zeolites; the main question is which of these routes is appropriate in terms of economic and environmental demands. The exploration of appropriate 
synthetic procedures for nanostructured materials with various particle shapes and sizes is important in nanotechnology and nanoscience. The development of strategies that reduce the use of SDAs or solvents, and time and energy consumption, while affording high yields is a priority.

\section{References}

[1] Perez-Ramirez J, Christensen C H, Egeblad K, et al. Hierarchical zeolites: enhanced utilisation of microporous crystals in catalysis by advances in materials design[J]. Chemical Society Reviews, 2008, 37(5):2530-42.

[2] Karwacki L, De Winter D A M, Aramburo L R, et al. Architecture-dependent distribution of mesopores in steamed zeolite crystals as visualized by FIB-SEM tomography.[J]. Angewandte Chemie, 2011, 123(6):1330-1334.

[3] Anbia M, Davijani A H. Synthesis of L-Cysteine grafted nanoporous carbon (CMK-3) and its use as a new cadmium sorbent[J]. Chemical Engineering Journal, 2013, 223(3):899-907.

[4] Marcilla A, Gómez-Siurana A, Berenguer D, et al. Reduction of tobacco smoke components yields by zeolites and synthesized Al-MCM-41[J]. Microporous \& Mesoporous Materials, 2012, 161(4):14-24.

[5] Liu B, Zheng Y, Hu N, et al. Synthesis of low-silica CHA zeolite chabazite in fluoride media without organic structural directing agents and zeolites[J]. Microporous \& Mesoporous Materials, 2014, 196(13):270-276.

[6] Perez-Ramirez J, Christensen C H, Egeblad K, et al. Hierarchical zeolites: enhanced utilisation of microporous crystals in catalysis by advances in materials design[J]. Chemical Society Reviews, 2008, 37(5):2530-42.

[7] Chal R, Gérardin C, Bulut M, et al. Overview and Industrial Assessment of Synthesis Strategies towards Zeolites with Mesopores[J]. Chemcatchem, 2011, 3(1):67-81.

[8] Sheng Q, Ling K, Li Z, et al. Effect of steam treatment on catalytic performance of HZSM-5 catalyst for ethanol dehydration to ethylene[J]. Fuel Processing Technology, 2013, 110(2):73-78.

[9] Hess C. Highly dispersed vanadium oxide catalysts[J]. Rsc Publishing, 2011.

[10] Möller K, Bein T. Mesoporosity-a new dimension for zeolites[J]. Chemical Society Reviews, 2013, 42(9):3689-707.

[11] Li K, Valla J, Garcia-Martinez J. ChemInform Abstract: Realizing the Commercial Potential of Hierarchical Zeolites: New Opportunities in Catalytic Cracking[J]. Cheminform, 2014, 45(18):46-66.

[12] Serrano D P, Sanz R, Pizarro P, et al. Narrowing the mesopore size distribution in hierarchical TS-1 zeolite by surfactant-assisted reorganization[J]. Microporous \& Mesoporous Materials, 2014, 189(5):71-82.

[13] Verboekend D, Vilé G, Pérezramírez J. Mesopore Formation in USY and Beta Zeolites by Base Leaching: Selection Criteria and Optimization of Pore-Directing Agents[J]. Crystal Growth \& Design, 2012, 12(12):3123-3132.

[14] Lysenko N D, Il'In V G, Yaremov P S. Structural and sorption characteristics of the products from zeolitization of sba-15 in the presence of tetraalkylammonium hydroxides[J]. Theoretical and Experimental Chemistry, 2011, 47(4):257-263.

[15] Guo Y P, Wang H J, Guo Y J, et al. Fabrication and characterization of hierarchical ZSM-5 zeolites by using organosilanes as additives[J]. Chemical Engineering Journal, 2011, 166(1):391-400. 
[16] Jacobsen C J H, Madsen C, Houzvicka J, et al. Mesoporous Zeolite Single Crystals[J]. Journal of the American Chemical Society, 2000, 122(29):7116-7117.

[17] Qiang L, Gang L, Sun H. Synthesis of hierarchical TS-1 with convenient separation and the application for the oxidative desulfurization of bulky and small reactants[J]. Fuel, 2014, 130(7):70-75.

[18] Yin C, Tian D, Xu M, et al. One-step synthesis of hierarchical mesoporous zeolite Beta microspheres from assembly of nanocrystals[J]. Journal of Colloid \& Interface Science, 2009, 50(19):4496-4504. 